\title{
Re: Comparison of Radical Prostatectomy Techniques: Open, Laparoscopic and Robotic Assisted
}

\author{
Rodrigo Frota, Burak Turna, Rodrigo Barros, Inderbir S. Gill \\ Section of Laparoscopic and Robotic Surgery, Glickman Urological Institute, Cleveland Clinic \\ Foundation, Cleveland, Ohio, USA
}

Int Braz J Urol. 2008; 34: 259-69

To the Editor,

This timely paper reviews the current status and respective roles of laparoscopic, robotically-assisted and open radical prostatectomy in the management of localized prostate cancer. While open radical prostatectomy remains the gold standard of treatment, a minimally-invasive approach has been available since 1997 in the form of laparoscopic radical prostatectomy. Minimally-invasive approaches to radical prostatectomy hope to duplicate the benefits of this approach seen with other procedures, including decreased patient blood loss and post-operative recovery time. The increased visualization, through digitally enhanced images that both magnify and illuminate the operative field, contributes greatly to the performance of this technically challenging procedure. However, laparoscopic surgery requires the acquisition of new anatomical perspectives, hand-eye coordination and the capacity to operate with limited tactile feedback and lack of 3-dimensional vision, all of which contributes to its undeniably steep learning curve. More recently, robotic systems have been used as an additional tool for the laparoscopic approach, with the hypothesis that they might improve the precision and accuracy of the anatomical dissection for the reasons outlined in the introduction of the current paper.

The authors concisely summarize the available contemporary literature, paying most attention to larger series from centers with established reputations in this field and with longer term follow-up. Criteria for comparison include operative, oncological and functional outcomes, as well as a pertinent discussion of financial considerations. Advantages of the minimally-invasive approaches are seen in generally lower operative blood loss, marginally decreased complication rates and shorter duration of catheterization. Analgesia requirements appear to be comparable and length of hospital stay often depends on more than simply the operative technique involved. Data concerning functional outcomes appears to be similar across the different techniques, but the authors rightly point out the difficulties comparing like with like in these studies, in terms of definitions of continence and potency and the use of validated questionnaires. The long term oncological efficacy of RRP is well studied but as yet limited long-term follow up is available for the minimally-invasive approaches. PSA progression-free survival appears comparable in the short to medium term, and what comparative studies exist show no significant differences in positive margin rates.

Our own unit recently published a direct comparison of robotic-assisted versus pure laparoscopic radical prostatectomy (1). No significant differences were observed between the pure laparoscopic and the robotic-assisted procedure with regard to operative time, operative blood loss, length of hospital stay or bladder catheterization. A higher transfusion rate was seen in the robotic-assisted group (9.8\%) compared to the pure laparoscopic group, though this finding has not been borne out in other similar studies $(2,3)$. No significant differences were seen in the rate of major 
complications between the 2 groups. The rate of margin positivity did not significantly differ between pure laparosocopy $(15.8 \%)$ and the robotic-assisted procedure $(19.5 \%)$. Our conclusion was that pure laparoscopic extra-peritoneal radical prostatectomy is equivalent to the robotic-assisted procedure in a centre experienced in laparoscopic techniques.

The current review is a welcome addition to the comparative literature regarding the status of minimally-invasive techniques against the well-established gold standard of open surgery. Tooher et al., in their comprehensive review of this topic, concluded that any conclusions that can be drawn from these comparisons are limited by the nature of the available data (4). Well performed, randomized, controlled trials are urgently required to provide stronger evidence when comparing these techniques. Sufficient followup and the use of internationally validated measures of functional outcomes are essential.

\section{REFERENCES}

1. Rozet F, Jaffe J, Braud G, Harmon J, Cathelineau X, Barret $\mathrm{E}$, et al.: A direct comparison of robotic assisted versus pure laparoscopic radical prostatectomy: a single institution experience. J Urol. 2007; 178: 47882.

2. Menon M, Shrivastava A, Tewari A: Laparoscopic radical prostatectomy: conventional and robotic. Urology. 2005; 66 (5 Suppl): 101-4.

3. Joseph JV, Vicente I, Madeb R, Erturk E, Patel HR: Robot-assisted vs pure laparoscopic radical prostatectomy: are there any differences? BJU Int. 2005; 96: 39-42.

4. Tooher R, Swindle P, Woo H, Miller J, Maddern G: Laparoscopic radical prostatectomy for localized prostate cancer: a systematic review of comparative studies. J Urol. 2006; 175: 2011-7.

\section{Dr. François Rozet \&} Dr. Gordon P. Smith Department of Urology, Institut Montsouris Université Paris Descartes 42, Bd Jourdan, 75014 Paris, France E-mail:francois.rozet@imm.fr 